\title{
Liquid-Phase Exfoliation of Biochars in Green Solvents and Correlation with Solvent Parameters
}

Juliana L. Vidal ${ }^{a}$ (jlvidal@mun.ca), Stephanie M. V. Gallant ${ }^{a}$ (smg561@mun.ca), Evan P. Connors ${ }^{a}$ (epc682@mun.ca), Douglas D. Richards ${ }^{b}$ (d.douglasrichards@gmail.com), Stephanie L. MacQuarrie ${ }^{a, b}$ (stephanie macquarrie@cbu.ca), and Francesca M. Kerton*a (fkerton@mun.ca)

a Department of Chemistry, Memorial University of Newfoundland, St. John's - NL, A1B 3X7, Canada, E-mail: fkerton@mun.ca

${ }^{\mathrm{b}}$ Department of Chemistry, Cape Breton University, Sydney - NS, B1P 6L2, Canada, E-mail: stephanie macquarrie@cbu.ca

\section{ABSTRACT}

Liquid-phase exfoliation (LPE) is a process frequently used to overcome the interactions between layers in layered materials to produce small sheets of material, with remarkable properties and high value applications. Materials are prepared via direct or indirect sonication in a solvent that must be able to effectively disperse and stabilize the sheets produced. Unfortunately, the preferred solvents for exfoliation processes are often toxic and possess several health risks. In this work, we show that LPE in greener solvents can be used to access nanostructures of biochar and further improve the applications of this renewable and bio-based material. Herein, pristine and oxidized biochars prepared from hardwood and softwood biomass waste (e.g. sludge, bark, and sawdust) are exfoliated in a range of solvents to allow the identification of benign alternatives that could afford highly concentrated dispersions. The majority of biochar nanostructures produced after exfoliation are stacked nanosheets containing between 2-8 layers (average $15 \mathrm{~nm}$ thickness). Correlations between effective LPE of biochar in solvents and different solvent parameters, including Kamlet-Taft, were established and allowed greener solvents to be used. Surface modification of biochars (e.g. via oxidation) has potential to increase their dispersibility in more benign solvents. LPE of oxidized biochars is more efficient in hydrogen-bond accepting solvents due to the increased concentration of carboxylic acid and alcohol functional groups on the surface of particles, when compared to nonfunctionalized biochars. Dispersions containing 0.20-0.75 mg/mL exfoliated oxidized biochar were obtained in solvents such as polyethylene glycols, glycerol formal and c-caprolactone. Moreover, LPE of pristine biochars in dimethyl carbonate, ethyl acetate, and solketal gave similar yields to more commonly used solvent for this process, N-methyl-2-pyrrolidone (NMP) a known reprotoxic molecule. 


\section{INTRODUCTION}

Biochar is a renewable carbon-based material produced through the thermal degradation of biomass under low supplies of oxygen using a technique known as pyrolysis. ${ }^{1}$ Pyrolysis of waste biomass is considered to be a cost-efficient and sustainable process, because carbon dioxide $\left(\mathrm{CO}_{2}\right)$ fixed by green plants during their photosynthesis is removed from the carbon cycle and can be stored for centuries in the form of biochar. ${ }^{1,2}$ Pyrolysis also prevents the further release of greenhouse gases (e.g. methane and $\mathrm{CO}_{2}$ ) produced during the decay of waste biomass at processing sites or on the forest floor. Therefore, large-scale applications of biochar could play a role in diminishing $\mathrm{CO}_{2}$ concentrations in the atmosphere and in mitigating climate change effects, as has been described previously. ${ }^{2,3}$ It is estimated that storing carbon in the form of biochar could potentially avoid the emission of $0.1-0.3$ billion tons of $\mathrm{CO}_{2}$ per year. ${ }^{2}$

The structure of biochar is known to be inherently amorphous (i.e. mostly sp ${ }^{3}$ carbons in an extended network) but also contains some crystalline areas composed of graphene-like sheets (i.e. $\mathrm{sp}^{2}$ carbons) cross-linked randomly..$^{1,3}$ Although the large-scale production and utilization of biochar offers several advantages, the application of this carbon-based material is limited due to the variability and complexity of its molecular level structure. This structure is dependent on the nature of the feedstock and pyrolysis conditions employed. ${ }^{3,4}$ As a consequence, this economic, environmentally benign, and easily produced carbon material has been used mainly for soil remediation and pollutant removal, ${ }^{1,3}$ whereas in our opinion there is significant scope for its application in higher value fields. Expansion of biochar use could be very important during society's transition towards a bio-based economy. To this end, we have recently reported the use of oxidized biochars as catalysts for the synthesis of cyclic carbonates. ${ }^{5}$ Another approach is exfoliation and this approach may provide access to a better understanding of the material's structure.

Layered materials, such as biochar, are solids that present strong in-plane chemical bonds and weak interactions between layers, such as van der Waals. Through exfoliation, an external force is introduced to weaken or break these van der Waals attractions, thus producing a single or a small number $(<10)$ of stacked monolayers with enhanced chemical and physical properties. ${ }^{6-8}$ Applications for new materials created via exfoliation methods are diverse and promising, including photovoltaic or thermoelectric devices, electrodes for supercapacitors or batteries, and in reinforcing or conductive polymer composites. ${ }^{9-15}$

The exfoliation of materials can be achieved using several techniques including chemical vapor deposition, intercalation, and micromechanical cleavage. However, drawbacks associated with scalability, high-cost, complexity, or sensitivity to ambient conditions limit the applications of these processes. ${ }^{16,17}$ To this end, liquid-phase exfoliation (LPE) was notably introduced to produce graphene from graphite, ${ }^{18}$ and has emerged as a powerful technique to overcome some of the limitations of other processing methods. During LPE, the bulk material is immersed in an appropriate solvent and directly or indirectly sonicated (Figure 1) to overcome interactions between layers. ${ }^{19}$ Sonochemical procedures are often seen as greener alternatives to those performed under classical conditions since they can save energy, generate less waste, and use less hazardous reagents. ${ }^{20}$

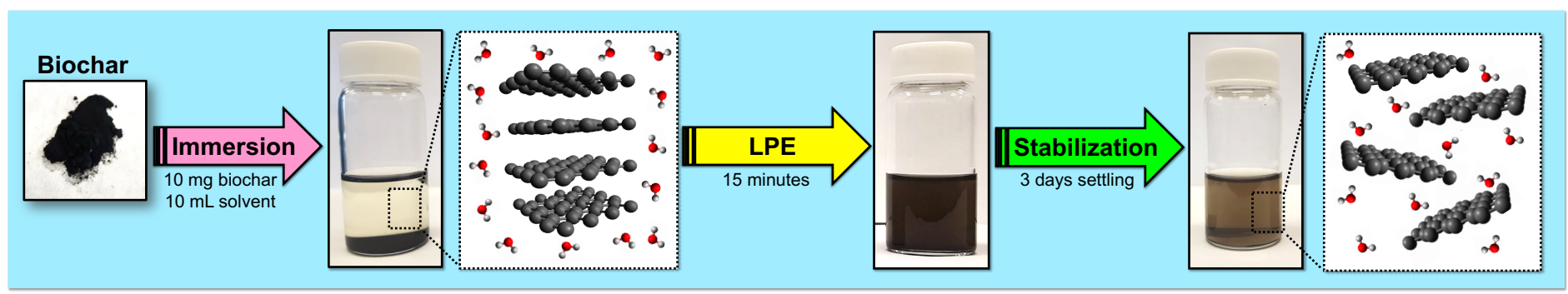

Figure 1 - Schematic diagram describing the liquid-phase exfoliation (LPE) process for biochar. Efficient and preferred solvents minimize the energy requirement of the process and stabilize the produced nanosheets. 
In the initial LPE work on graphite,$^{18}$ the amount of carbonaceous material dispersed in the solvent system was shown to be directly related to the densities $(\rho)$ and surface tensions $(\gamma)$ of the solvents used. It was proposed that ideal values of $\rho$ and $\gamma$ could decrease the necessary energy to separate the nanosheets and stabilize the exfoliated material against reaggregation. ${ }^{16-18,21}$ However, this surface-matching aspect alone could not explain some of the divergences observed in the exfoliation of other materials. For those reasons, solvent parameters such as the Hildebrand and Hansen solubility parameters, which can evaluate the interactions between solvent and material, were used to aid in the explanation of the liquid-phase processing in later studies. ${ }^{22,} 23$ Information that can be obtained from Hildebrand solubility parameters is somewhat limited because they only apply to nonpolar systems. ${ }^{24}$ Hansen solubility parameters (HSP) have been widely used to study the solubility of polymers in different solvents, but even those cannot fully describe or predict the best solvents for LPE. 8, 22, 23, 25 Kamlet-Taft solvatochromic parameters are considered the most extensive and useful parameters for the investigation and understanding of solvation effects, ${ }^{26,27}$ and yet as far as we are aware, they have never been used to describe the interaction between solvents and nanostructures of relevance to LPE processes. Therefore, the nature of molecular level solvent-nanostructure interactions such as hydrogen-bonding, that would surely play an important role in stabilizing nanosheets, is currently unclear.

With respect to biochar, exfoliation has been achieved using LPE, ${ }^{28-31}$ as well as mechanochemical techniques, ${ }^{32,33}$ and chemical pre-treatment of biomass. ${ }^{34,}{ }^{35}$ Although LPE may be the cheapest and most scalable biochar exfoliation technique due to the use of mild conditions, it cannot be considered the most benign to human health and the environment. Preferred solvents for the LPE of biochar possess several health risks, and a comprehensive study to identify highperformance solvents for the exfoliation of this bio-based material has not been performed. Only preliminary assessments regarding solvent parameters and their influence on exfoliation of biochar have been made, and samples have been typically

exfoliated in a reproductive toxicant solvent ( $N$-methyl-2-pyrrolidone) or processed in water with very low yields. ${ }^{28-31}$ An increased knowledge regarding solvent properties, biomass feedstock, and biochar functionality related to the efficiency of exfoliation can further improve our understanding of biochar's structure, promote the study of new sustainable and effective alternative solvents for LPE, and improve biochar applications as an environmentally benign advanced material. For those reasons, we herein describe an in-depth investigation of the liquid-phase exfoliation of biochar. Besides the comprehension of solvent and surface-matching effects that can greatly influence the process, we also identified more benign solvents for the production of nanosheets of this carbon material, which have large potential to be applied in high value-ended fields.

\section{EXPERIMENTAL}

Synthesis and characterization of pristine and functionalized biochars. Pristine biochars (bc) were prepared from hardwood and softwood residues as described previously. ${ }^{36}$ The oxidized analogues (ox-bc) were obtained after reaction of bc $(3.0 \mathrm{~g})$ with nitric acid $(7 \mathrm{~mol} / \mathrm{L})$ at $90^{\circ} \mathrm{C}$ for $3 \mathrm{~h}$. Samples were washed with deionized water until neutral $\mathrm{pH}$ and then dried in the oven at $100^{\circ} \mathrm{C}$ overnight. Materials were characterized via IR spectroscopy, thermogravimetric analysis (TGA), and elemental analysis (EA) has been reported in our previous work. To further assess surface functionality, Boehm titrations were performed, ${ }^{37,38}$ showing an increase in the number of acidic sites (nCSF) (from $3.84 \mathrm{mmol} / \mathrm{g}$ in bc to 4.88 $\mathrm{mmol} / \mathrm{g}$ in ox-bc).

Biochar exfoliation procedure. $10 \mathrm{~mL}$ of a solvent (purchased from Sigma-Aldrich or Fischer Scientific) was added to a vial containing $10 \mathrm{mg}$ of pristine or oxidized biochar from different biomass feedstocks, producing light gray dispersions. Samples were then directly sonicated for 15 min using a Misonix S-4000 Sonicator (containing a generator to supply the electrical signal, an aluminum transducer to convert the electrical signal to a mechanical vibration, a titanium alloy probe to transmit the vibration) in a sound enclosure. A circulating-cooling bath at $3{ }^{\circ} \mathrm{C}$ was used to prevent heating of samples. A 
schematic representation of the sonication setup used can be found in Figure S1, Supporting Information. An ultrasonic vibration amplitude of $50 \%$ and power of $20 \mathrm{~W}$ was chosen to perform the exfoliations, providing different energies depending on the solvent used. Solvents used for this procedure included, with abbreviation and the energy in parenthesis: acetone $(E=17,400 \mathrm{~J})$; benzonitrile $(\mathrm{PhCN}, \mathrm{E}=23,800 \mathrm{~J})$; 1-butanol $(\mathrm{E}=40,500 \mathrm{~J})$; $\varepsilon$-caprolactone $(\varepsilon-\mathrm{CL}, \mathrm{E}=36,800 \mathrm{~J})$; chloroform $\left(\mathrm{CHCl}_{3}, \mathrm{E}=18,800 \mathrm{~J}\right)$; cyclohexanone $(E=42,100 \mathrm{~J})$; Cyrene ${ }^{\circledR}(E=48,200 \mathrm{~J})$; 1,2-dichlorobenzene $(1,2-\mathrm{DCB}$, $\mathrm{E}=29,500 \mathrm{~J})$; dichloromethane $\left(\mathrm{CH}_{2} \mathrm{Cl}_{2}, \mathrm{E}=14,000 \mathrm{~J}\right)$; dimethyl carbonate $(\mathrm{DMC}, \mathrm{E}=25,800 \mathrm{~J})$; $D$-limonene $(\mathrm{E}=24,800$ $\mathrm{J}) ; \mathrm{N}, \mathrm{N}$-dimethylacetamide (DMA, E = 20,200 J); N,N-dimethylformamide (DMF, E = 20, $600 \mathrm{~J}$ ); dimethyl sulfoxide (DMSO, $E=33,4000 \mathrm{~J})$; ethanol $(E=25,200 \mathrm{~J})$; ethylene glycol $(E G, E=47,100 \mathrm{~J})$; ethyl acetate $(E t O A c, E=20,500 \mathrm{~J})$; ethyl lactate $(E=28,800 \mathrm{~J})$; glycerol formal $(G F, E=38,300 \mathrm{~J})$; hexafluorobenzene $\left(\mathrm{C}_{6} \mathrm{~F}_{6}, E=20,300 \mathrm{~J}\right)$; 4-methyl-2-pentanone $(E=$ 19,600 J); N-methyl-2-pyrrolidone (NMP, E = 31,500 J); polyethylene glycol 200 (PEG 200, E = 43,600 J); polyethylene glycol 400 (PEG 400, E = 50,200 J); solketal ( $=$ 42,700 J); toluene ( $=20,900 \mathrm{~J})$; and deionized water $(E=25,800 \mathrm{~J})$. After sonication, black dispersions of biochar were obtained for most of the systems studied. However, only a few solvents led to dispersions with significant concentrations of biochar nanostructures after allowing unstabilized materials to precipitate. The samples were allowed to settle for 3 days at ambient conditions, the dispersed fraction was carefully separated for further characterization, and $w t \%$ dispersed determined via UV-Vis analysis. The dispersions were stable for at least 3 weeks.

Raman spectroscopy. Analyses were performed using a Renishaw confocal Raman microscope with an $830 \mathrm{~nm}$ wavelength laser. Biochar samples before exfoliation were mounted on a quartz wafer, whereas exfoliated samples were mounted on a silicon wafer after solvent drying. Scans were performed at $0.5 \%$ or $1 \%$ laser power for 20 or 25 seconds, using 20x optical lens. Baseline correction was implemented using a cubic spline interpolation smoothing in Renishaw's WiRE software, while peak areas and heights were calculated using a Gaussian fit in IGOR Pro software.

Atomic Force Microscopy (AFM). AFM characterization of exfoliated biochars was performed using an MFP-3D Asylum Research instrument and NSC35/AI BS tips by MikroMasch. Samples were drop-cast onto a glass slide pre-heated at 100 ${ }^{\circ} \mathrm{C}$ to avoid reaggregation of the nanostructures and promote a more uniform deposition. Samples were scanned at $0.1 \mathrm{~Hz}$. The heights of 100 different nanostructures were calculated to obtain a distribution of their thicknesses.

Transmission Electron Microscopy (TEM). TEM characterization of biochars before and after exfoliation in NMP were carried out at Cape Breton University, Sydney-NS, using a Hitachi HT7700 Transmission Electron Microscope containing a tungsten filament in high contract $(\mathrm{HC})$ mode at $80 \mathrm{kV}$.

Mass spectrometry (MS). Data were acquired using a Matrix Assisted Laser Desorption/lonization-Time of Flight (MALDITOF) Bruker UltrafleXtreme MS in positive-ion mode. About $1.2 \mathrm{mg}$ of pristine or oxidized biochar samples exfoliated in EtOAc were dissolved in $50 \mu \mathrm{L}$ of a $1 \mathrm{mg} / \mathrm{mL}$ standard solution of 2,5-dihydroxybenzoic acid (DHB) in tetrahydrofuran (THF). After centrifugation, $0.5 \mu \mathrm{L}$ of the supernatant of the mixture was deposited on the MALDI plate. During analysis, the laser power was carefully increased to $90 \%$ to obtain the best results.

\section{RESULTS AND DISCUSSION}

Effects of solvent parameters in the exfoliation of biochars. To begin our studies, pristine (bc) and oxidized biochars (ox-bc) from different biomass feedstocks were exfoliated in a wide range of different solvents (Figure S2, Supporting Information). After settling, the concentration and percentage of biochar dispersed in the solvent was measured using UVVis spectroscopy at $660 \mathrm{~nm}$ (Figure S3, Supporting Information). The molar absorption coefficient $(\alpha)$ was calculated 
using the Beer-Lambert law (Figure S4, Supporting Information). The slope of the graph of absorbance divided by cell length $(A / l)$ as a function of biochar wt\% gives a value of $\alpha=422 \mathrm{~L} \mathrm{~g}^{-1} \mathrm{~m}^{-1}$, which is lower than in some other works involving the exfoliation of carbon materials, ${ }^{18,39}$ but comparable to a study involving graphite exfoliation using the same processing time as used herein (15 min sonication). ${ }^{40}$

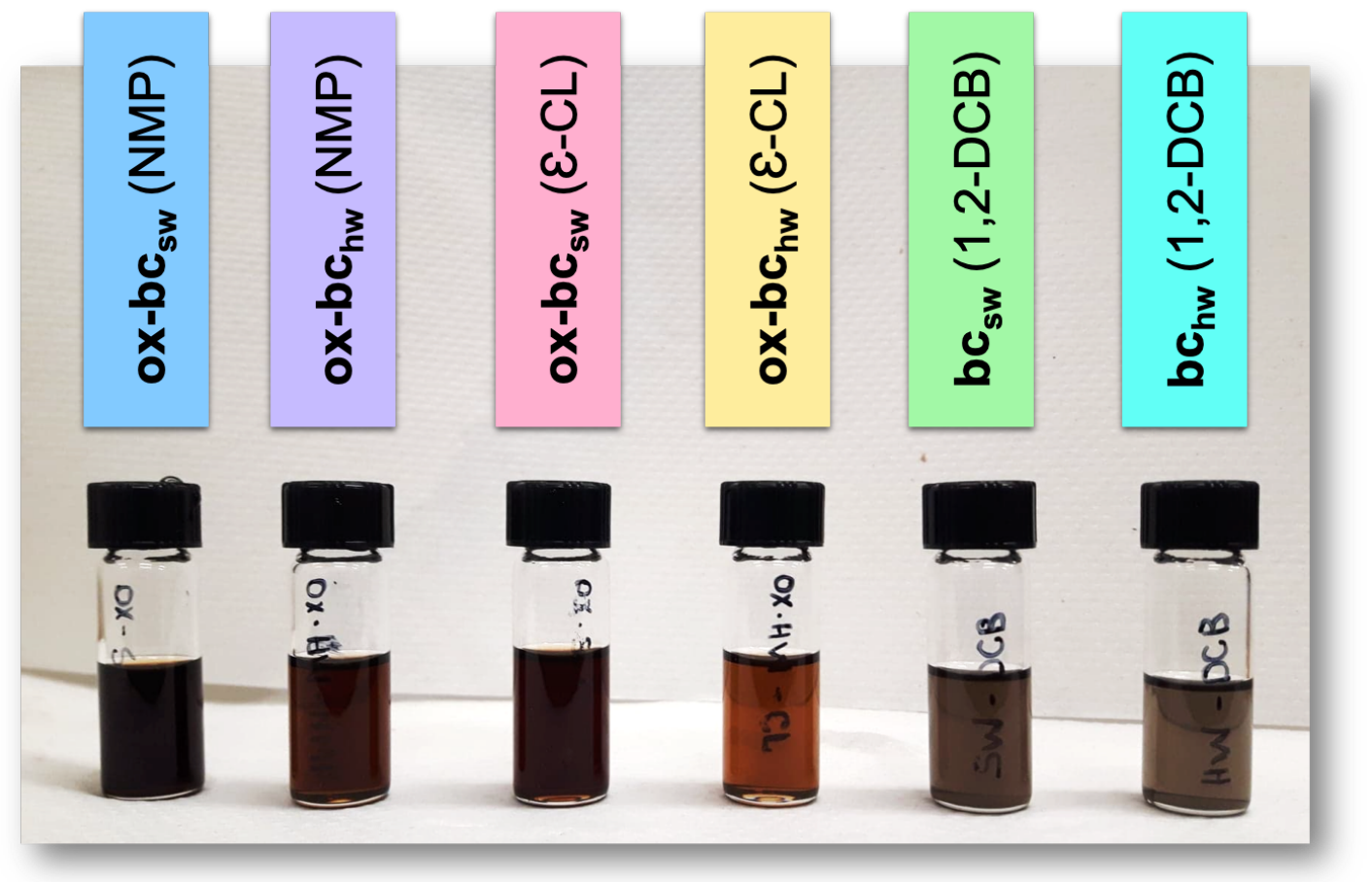

Figure 2 - Visual comparison of biochar dispersions from different feedstocks, oxidized and pristine, in a range of solvents.

To identify some of the most effective solvents for LPE of biochar, we first evaluated the surface-matching aspect of the solvents in the process, as this has been critical for other layered materials reported in the literature. We observed that the best solvents for dispersing pristine biochars from hardwood $\left(\mathbf{b c}_{\mathbf{h w}}\right)$ and softwood $\left(\mathbf{b c}_{\mathbf{s w}}\right)$ biomass had density values of $\rho \sim 1.0 \mathrm{~g} / \mathrm{mL}$ and surface tension values of $\gamma \sim 20 \mathrm{mN} / \mathrm{m}$. Some examples of the solvents used in the preliminary studies include chloroform $\left(\mathrm{CHCl}_{3}\right)$, dichloromethane $\left(\mathrm{CH}_{2} \mathrm{Cl}_{2}\right)$, and 1,2-dichorobenzene (1,2-DCB). Although density values of $\rho \sim$ $1.0 \mathrm{~g} / \mathrm{mL}$ were shown to lead to an effective exfoliation of the material, extremely high values were found to be detrimental to the process. When hexafluorobenzene $\left(\mathrm{C}_{6} \mathrm{~F}_{6}\right)$, the solvent with the highest density studied was used $(1.62 \mathrm{~g} / \mathrm{mL})$, bc tended to stay on the surface forming a thin film, instead of being dispersed in the medium. This also happened when $\mathrm{CHCl}_{3}$ was used to disperse $\mathbf{b} \mathbf{c}_{\mathrm{hw}}$, but $\mathbf{b} \mathbf{c}_{\mathrm{sw}}$ could be dispersed in $\mathrm{CHCl}_{3}$ and these data suggest that the two bc must possess different chemical and physical properties, including different surface functionalities.

For the oxidized biochars from hardwood (ox-bchw) and softwood (ox-bcsw) biomasses, the amount of solid dispersed in the best solvents for bc exfoliation (e.g. $\mathrm{CHCl}_{3}, \mathrm{CH}_{2} \mathrm{Cl}_{2}$, and 1,2-DCB) was negligible (< $0.05 \mathrm{mg} / \mathrm{mL}$ ). The highest levels of exfoliated ox-bc samples were obtained in solvents with density values of $\rho \sim 1.0 \mathrm{~g} / \mathrm{mL}$ and surface tension values of $\gamma \sim$ $40 \mathrm{mN} / \mathrm{m}$, such as $\mathrm{N}$-methyl-2-pyrrolidone (NMP) and dimethyl sulfoxide (DMSO). These results demonstrated that different functional groups play a critical role in aiding dispersion of these functional biochars compared with the pristine analogues. It is worth mentioning that the solvents screened initially focused on those employed in previous literature LPE studies and did not consider environmental toxicity and related factors, as most of these solvents are not environmentally benign in nature. 
During our studies, we observed that lower levels of dispersion were achieved for $\mathbf{b c h}_{\mathrm{hw}}$ and $\mathbf{o x}-\mathbf{b} \mathbf{c}_{\mathrm{hw}}$, when compared to $\mathbf{b c}_{\mathbf{s w}}$ and $\mathbf{o x}-\mathbf{b} \mathbf{c}_{\mathbf{s w}}$. This difference can be seen visually in Figure $\mathbf{2}$ and in the data from Tables S1 and S2 (Supporting Information). This particular difference was surprising, because the same biochars from different waste biomass feedstocks showed very similar behaviors and gave similar results when applied as catalysts to produce cyclic carbonates in our previous work. ${ }^{5}$ To explain the unpredicted differences observed herein, the densities of $\mathbf{b c}_{\mathbf{s w}}$ and $\mathbf{b} \mathbf{c}_{\mathrm{hw}}$ were calculated using a pycnometer. The density of $\mathbf{b c}_{\mathrm{hw}}$ was found to be lower $(1.02 \mathrm{~g} / \mathrm{mL})$ than $\mathbf{b c}_{\mathbf{s w}}(1.19 \mathrm{~g} / \mathrm{mL})$, which could help to understand the observed difference in the exfoliation of these materials. Besides their density, the molecular structure of biochars, such as the relative amounts of $\mathrm{sp}^{2}$ and $\mathrm{sp}^{3}$ carbons, and amount of residual oxygen from the original lignocellulosic biomass could also have an impact in the dispersion levels achieved and was therefore investigated prior to identifying greener solvents for the LPE process.

Characterization of exfoliated biochars. To aid in understanding the molecular structure of biochars, the LPE effects on them, and the differences observed between biochars from different feedstocks, Raman spectroscopy experiments were performed on biochars before and after exfoliation. $\mathrm{CHCl}_{3}$ was chosen as a solvent for Raman analysis due to its high exfoliation efficiency for pristine samples and low boiling point, thus providing fast evaporation on the silicon wafer used as a support. As shown in Figure 3, the Raman spectra of as prepared and exfoliated pristine biochars contain two bands, known as $G$ and $D$ bands.

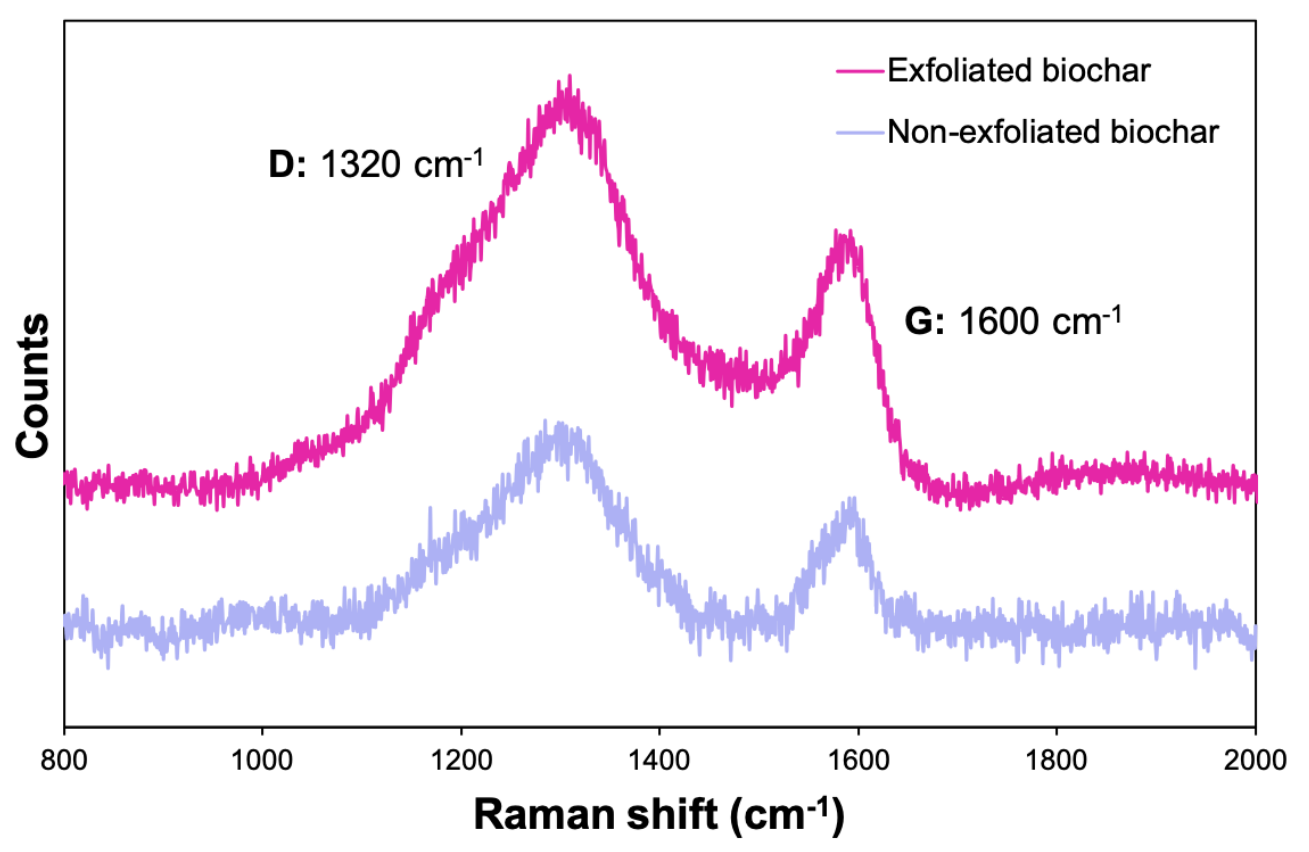

Figure 3 - Raman spectra of $\mathbf{b c}_{\mathrm{hw}}$ before and after exfoliation. The $\mathrm{D}$ and $\mathrm{G}$ bands are related to the crystallinity and deformities of the material produced and can be used to estimate the effect of exfoliation on its chemical structure.

The $\mathrm{G}$ band (observed at $1600 \mathrm{~cm}^{-1}$ ) is produced from the stretching vibration of $\mathrm{sp}^{2}$ carbons, whilst the $\mathrm{D}$ band (observed at $1320 \mathrm{~cm}^{-1}$ ) originates from the breathing mode of hexagonal rings and it is only Raman active if the ring is close to a defect. ${ }^{41,42}$ Therefore, the $G$ band indicates crystallinity or presence of graphitic arrangements, whereas the $D$ band is related to defects or disorders of the material. Although the Raman spectra of natural and exfoliated hardwood biochar samples are very similar, the ratio between the intensity of these two bands $\left(I_{G} / l_{D}\right)$ is different. The $I_{G} / l_{D}$ ratio is known to estimate the molecular order within carbon network. ${ }^{43}$ In the current study, as-prepared and exfoliated hardwood 
biochar samples present a higher $I_{G} / l_{D}$ ratio when compared to the softwood analogues, suggesting an increased $s p^{2}$ carbon content and higher level of crystallinity (Table 1).

Table 1 - Exfoliation effects in the molecular structure of biochars from different biomass sources, determined by Raman spectroscopy.

\begin{tabular}{|c|c|c|}
\hline Biochar type & $I_{G} / I_{D}$ (non-exfoliated) & $I_{G} / I_{D}$ (exfoliated) \\
\hline bchw & $0.45 \pm 0.01$ & $0.55 \pm 0.05$ \\
\hline $\mathrm{bc}_{\mathrm{sw}}$ & $0.18 \pm 0.06$ & $0.19 \pm 0.01$ \\
\hline
\end{tabular}

Because graphitic structures are more ordered, dense, and rigid than the amorphous phase, they are less susceptible to exfoliation, which explains the increased wt\% dispersions obtained via LPE for exfoliated softwood biochar in all the solvents studied and the difficulty in finding high-performance solvents for the processing of the more crystalline materials. Although density filters were used during Raman analysis to decrease the laser power and avoid potential thermal degradation of samples, the spectra of ox-bc before and after exfoliation could not be obtained. We presume that, due to the existence of local functional differences and greater molecular asymmetry, bonding vibrations and rotations in ox-bc might not be intense enough to provide signals or may lead to Raman inactive vibrations.

Raman spectroscopy has also been previously used to estimate the crystallite size ( $\left.L_{a}\right)$, distance between defects ( $\left.L_{D}\right)$ and number of defects $\left(n_{D}\right)$ of graphene related systems. ${ }^{43,44}$ This estimation can be obtained by equations that relate the $I_{G} / I_{D}$ ratios of the compounds with Raman laser energy (Equations S1-S3, Supporting Information). Applying the same strategy to biochar (Table 2), we observe an increased $L_{a}$, longer $L_{D}$ and smaller $n_{D}$ in the exfoliated samples, when compared to the non-exfoliated ones. $L_{a}$ values obtained herein are higher than ones obtained for exfoliated multiheteroatom co-doped biochars (i.e. $5 \mathrm{~nm}$ ), ${ }^{31}$ but lower than values obtained for LPE of graphite in NMP (i.e $136 \mathrm{~nm}$ ) in a previous work using the same estimation method herein. ${ }^{40}$ In comparison with the exfoliated biochar, graphene samples also present lower values of $L_{D}$ (i.e. $31.6 \mathrm{~nm}$ ) and $\mathrm{n}_{D}\left(\right.$ i.e. $3.6 \times 10^{-10} \mathrm{~cm}^{-2}$ ) due to the presence of a more ordered, crystalline, and organized chemical structure. ${ }^{40}$

Table 2 - Exfoliation effects in the estimation of crystallite size $\left(\mathrm{L}_{\mathrm{a}}\right)$, distance between defects $\left(\mathrm{L}_{\mathrm{D}}\right)$, and number of defects ( $\left.\mathrm{n}_{\mathrm{D}}\right)$ of biochars from different biomass sources, determined by Raman spectroscopy.

\begin{tabular}{llll}
\hline Biochar type & La $(\mathbf{n m})$ & Lo $(\mathbf{n m})$ & no $\left(\mathbf{c m}^{-2}\right)$ \\
\hline Non-exfoliated bchw & 51.2 & 19.6 & $8.43 \times 10^{10}$ \\
Exfoliated bchw & 62.6 & 21.7 & $6.90 \times 10^{10}$ \\
Non-exfoliated bc $c_{\mathrm{sw}}$ & 20.5 & 12.4 & $2.11 \times 10^{11}$ \\
Exfoliated bc & 21.6 & 12.7 & $2.00 \times 10^{11}$ \\
\hline
\end{tabular}

More insights regarding the effect of exfoliation upon the structure of biochar could be obtained via AFM. Biochar samples after exfoliation in $\mathrm{CHCl}_{3}$ were drop-cast on a glass slide (pre-heated to $100{ }^{\circ} \mathrm{C}$ before analysis to promote a uniform dispersion and avoid the reaggregation of nanosheets as described in previous work). ${ }^{17}$ Herein, 100 nanostructures of exfoliated $\mathbf{b c}_{\mathrm{hw}}$ observed in different AFM images (examples in Figure 4A-D) were randomly selected and their thicknesses measured to yield a distribution (Figure 4E). The lowest height value measured was found to be $15 \mathrm{~nm}$, which can be attributed to the thickness of an individual biochar nanosheet. This value can be divided by the distance between defects obtained via Raman $\left(L_{D}\right)$ to give 1 as the approximate number of exfoliated biochar layers $(N)$. From Figure 4E, the 
majority of nanostructures (about $60 \%$ ) are $15-120 \mathrm{~nm}$ thick, thus confirming the predominance of single to a few multilayered (i.e. $N$ values in the range of 2-8) biochar sheets produced after exfoliation.
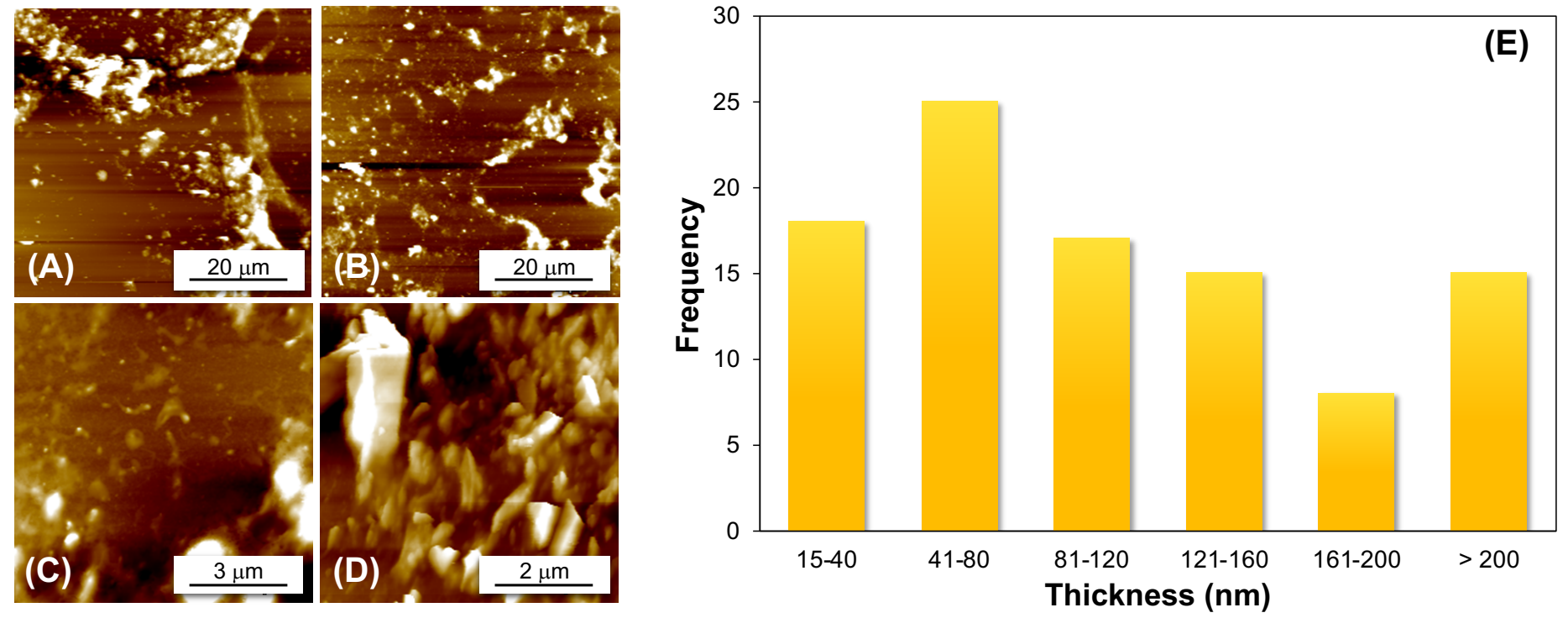

Figure 4 - AFM characterization of exfoliated biochar samples. Figures (A-D) show the AFM images of exfoliated bchw samples used to calculate the number of nanostructures containing different heights. The correlation between frequency and exfoliated material thickness can be observed in Figure (E).

TEM micrographs of pristine hardwood samples before and after exfoliation in NMP are presented in Figure 5. A multilayered structure of biochar is seen in samples of $\mathbf{b c}_{\mathrm{hw}}$ before exfoliation (Figure 5A and Figure 5B), whereas nanocrystalline structures are present in the exfoliated biochar. Micrographs of the processed material show the presence of aromatic clusters black dots with average length of $18 \pm 4 \mathrm{~nm}$ (Figure 5C). Similar structures have been assigned to multi-layered graphene-like nanosheets randomly ordered. ${ }^{28}$ Nanocrystalline stripes could also be observed in the structure of exfoliated biochar, as well as the presence of atomic arrangements at the edges of the dispersed material (Figure 5D). The presence of aromatic clusters, nanocrystalline stripes, and atomic arrangements in the structure of exfoliated biochar observed via TEM demonstrate the value of LPE in gaining access to the nanostructures of this bio-renewable material.

Although the presence of distinct single layers of biochar are not observed via TEM due to nanosheet reaggregation, which is a reoccurring problem during the characterization of nanosheets obtained during LPE, the morphology and structures visible herein by TEM for $\mathbf{b c}_{\mathbf{h w}}$ and $\mathbf{b} \mathbf{c}_{\mathbf{s w}}$ are similar to those seen previously for rice straw biochar. ${ }^{28}$ TEM micrographs of the remaining biochars studied (ox-bchw, ox-bc $\mathbf{c}_{\mathbf{s w}}$, and $\mathbf{b} \mathbf{c}_{\mathbf{s w}}$ ) before and after treatment using ultrasound are shown in Figure S5 (Supporting Information). Exfoliated materials from pristine softwood precursors (Figure S5B, Supporting Information) show smaller aromatic clusters (i.e. average length of $10 \pm 3 \mathrm{~nm}$ ) when compared to hardwood samples. This may be related to the lower intrinsic crystallinity of $\mathbf{b c}_{\mathbf{s w}}$ indicated by Raman spectroscopy. The oxidation of biochar itself can weaken interlayer interactions within the sample due to the insertion of a large number of oxygencontaining groups, and might lead to a larger number of dispersed species after exfoliation of ox-bchw and $\mathbf{o x}_{\mathbf{b}} \mathbf{b} \mathbf{c}_{\mathbf{s w}}$ (Figure S5D and S5F, Supporting Information). Nevertheless, the sizes seen for ox-bc are very similar to the pristine exfoliated samples (i.e. average length of $17 \pm 4 \mathrm{~nm}$ for $\mathbf{o x}-\mathbf{b} \mathbf{c}_{\mathrm{hw}}$ and average length of $12 \pm 3 \mathrm{~nm}$ for $\mathbf{o x}-\mathbf{b} \mathbf{c}_{\mathbf{s w}}$ ). 

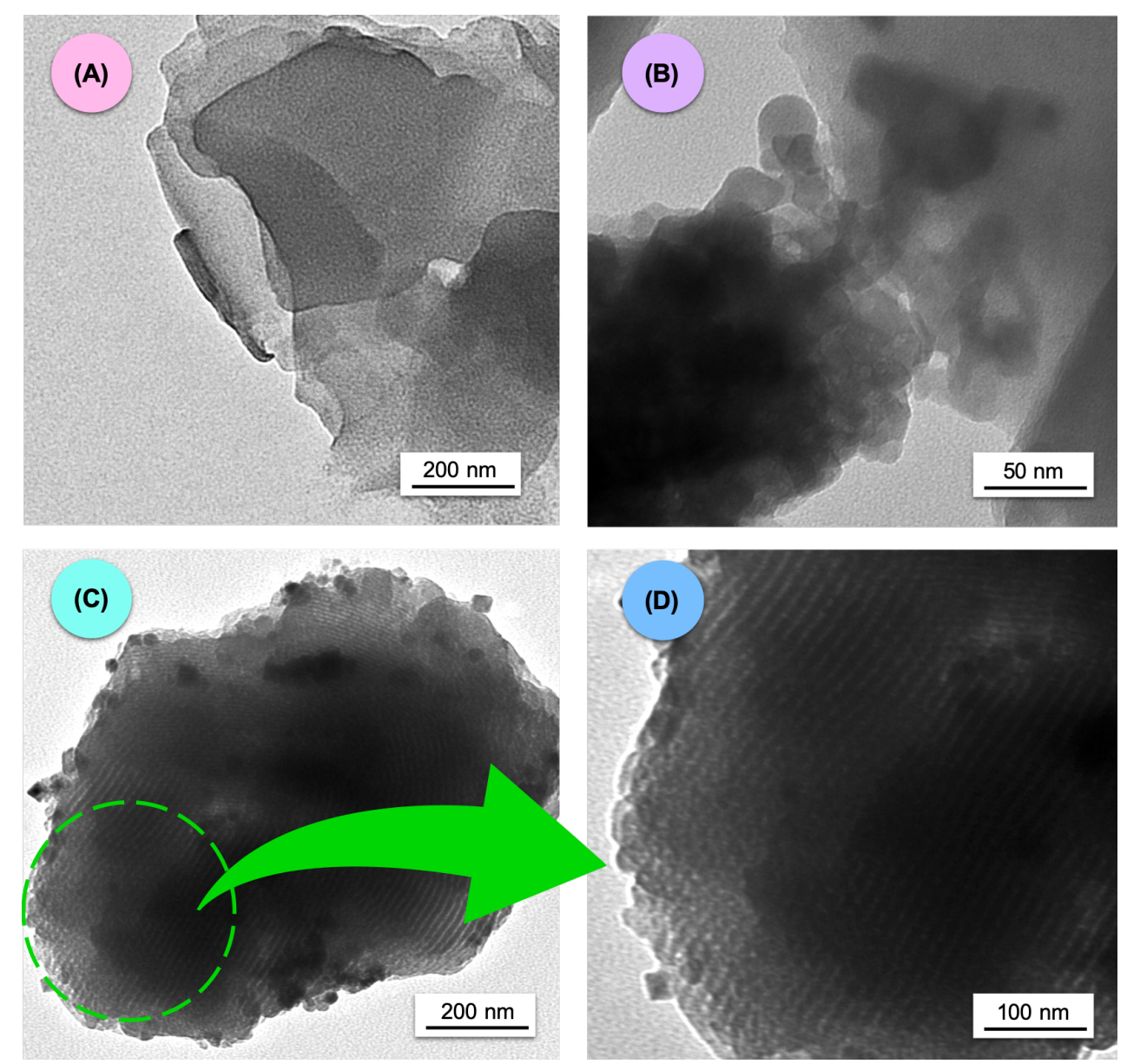

(D)

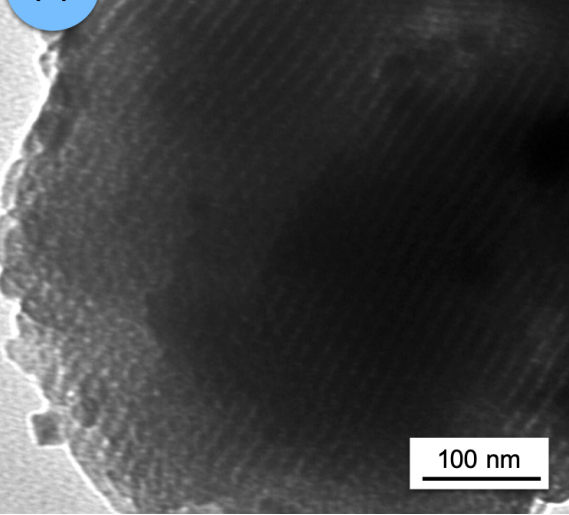

Figure 5 - TEM micrographs of hardwood biochar $\left(\mathbf{b c h}_{\mathrm{hw}}\right)$ samples in NMP. Figures $(A)$ and $(B)$ show the layered structure of bchw before exfoliation, whereas aromatic clusters (i.e. black dots) and nanocrystalline stripes are observed after biochar exfoliation in Figure (C). Atomic arrangements can be observed at the edges of the exfoliated material and are further highlighted in Figure (D).

In order to better understand the molecular structure of exfoliated biochars observed in the TEM micrographs, MS analyses were performed on $\mathbf{b} \mathbf{c}_{\mathrm{hw}}$ and $\mathbf{o x}-\mathbf{b} \mathbf{c}_{\mathrm{hw}}$ after exfoliation. As shown in Figure 6, a signal with $\mathrm{m} / \mathrm{z} 854.50 \mathrm{Da}$ is observed for exfoliated $\mathbf{b c h}_{\mathrm{hw}}$ and hardly any low intensity signals are seen at masses higher than this. For exfoliated ox-

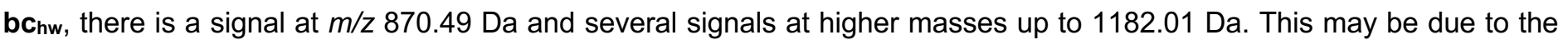
range of high molecular weight oxygen-containing species in the oxidized biochar samples, which have a greater tendency to interact with the organic acid matrix used in the analysis. As expected, the spectra do not represent a single molecular structure, the exfoliated samples seem to display a wide range of structures with primarily 16 and 72 Da weight differences between peaks. These masses can be assigned to an oxygen atom and six carbons (from the aromatic structures), respectively. In comparison with the unexfoliated analogue (Figure S6, Supporting Information), the MALDI spectrum of exfoliated $\mathbf{b c}_{\mathrm{hw}}$ is less complex (e.g. fewer peaks) and this may result from a fractionation of the carbon materials upon exfoliation with only lower mass species becoming dispersed. Further experiments would be needed to gain improved insight into the mass data obtained. 

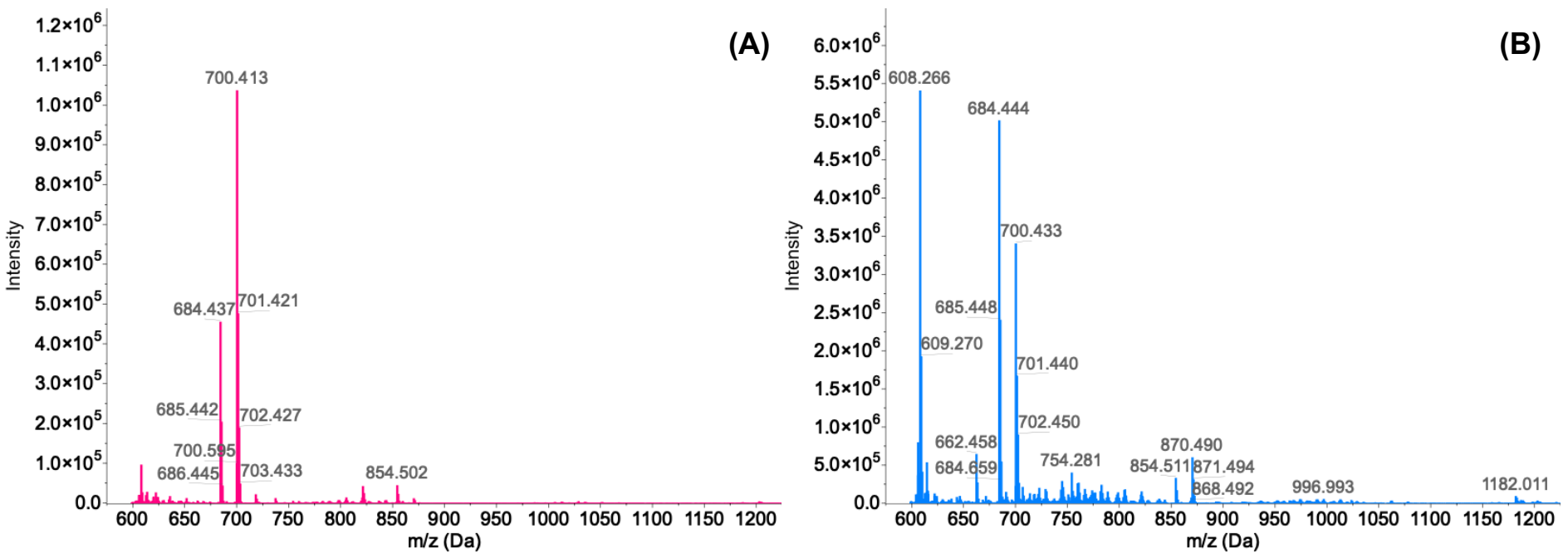

Figure 6 - MALDI-TOF spectra of exfoliated (A) $\mathbf{b} \mathbf{c}_{\mathrm{hw}}$ and $(\mathrm{B}) \mathbf{o x}-\mathbf{b} \mathbf{c}_{\mathrm{hw}}$ samples in positive-ion mode.

Green solvents for biochar exfoliation. Based on our preliminary studies that identified the ideal values of surface tension $(\gamma)$ and density $(\rho)$ of solvents for LPE of pristine (bc) and oxidized (ox-bc) biochars, an attempt to explore the nature of biochar exfoliation and an assessment regarding greener solvents for processing was realized. In Tables S1 and S2 (Supporting Information), a correlation between solvent parameters and percentage of bc and ox-bc dispersed in the samples during LPE can be seen. Reproducibility was assessed by performing exfoliations in triplicate. As expected, the evaluation of LPE using only the surface-matching aspect (i.e. density and surface tension) was superficial, and contributions from biochar-solvent interactions are also needed. Aware of the well-known limitations of Hildebrand's solubility parameters (e.g. only applicable for nonpolar systems), Hansen solubility parameters (HSP) and their effects in biochar exfoliation were assessed. HSP have been extensively used to aid in the selection of solvents in coating industries and to predict solvent-polymer compatibility. ${ }^{22,45}$ Each substance can be described using three HSP, which are related to the energy from dispersions forces $(\delta \mathrm{D})$, dipolar intermolecular forces $(\delta \mathrm{P})$, and hydrogen bonds $\left(\delta_{H}\right)$ between molecules. ${ }^{45}$ When HSP of the solvent and solute are similar, they usually show a high affinity for each other and are likely to mix easily to form a solution. One might expect similarities between the solvent and materials' surface functionality to aid in dispersion processes. Some of the solvents able to disperse bc and ox-bc had HSP values in the following ranges: $\delta_{\mathrm{D}} \sim 17 \mathrm{MPa}^{1 / 2}, \delta_{\mathrm{H}}$ $\sim 7 \mathrm{MPa}^{1 / 2}, \delta_{\mathrm{P}} \sim 6 \mathrm{MPa}^{1 / 2}$ (average values for dichloromethane and ethyl acetate) and $\delta_{\mathrm{D}} \sim 17 \mathrm{MPa}^{1 / 2}, \delta_{\mathrm{H}} \sim 20 \mathrm{MPa}^{1 / 2}, \delta_{\mathrm{P}} \sim$ $11 \mathrm{MPa}^{1 / 2}$ (average values for glycerol formal and ethylene glycol). However, some solvents with HSP values out of the ideal ranges were also able to exfoliate bc and ox-bc. For example, $\delta_{H}=0$ and $\delta_{\mathrm{P}}=0$ for $\mathrm{C}_{6} \mathrm{~F}_{6}$ which was an effective solvent in bc LPE. Other examples of effective LPE solvents outside the two typical HSP ranges included PhCN, DMC, solketal, PEGs 200 and 400, and $\varepsilon-C L$ (Tables S1 and S2, Supporting Information). Therefore, even though HSP were used by others previously to evaluate the nature of exfoliation of materials, ${ }^{39,40}$ they do not effectively describe (or predict) the LPE of biochar because a direct, regular, and predictable relationship between the high-performance solvents for biochar exfoliation and $\delta_{\mathrm{D}}, \delta_{\mathrm{H}}, \delta_{\mathrm{P}}$ values could not be obtained. 


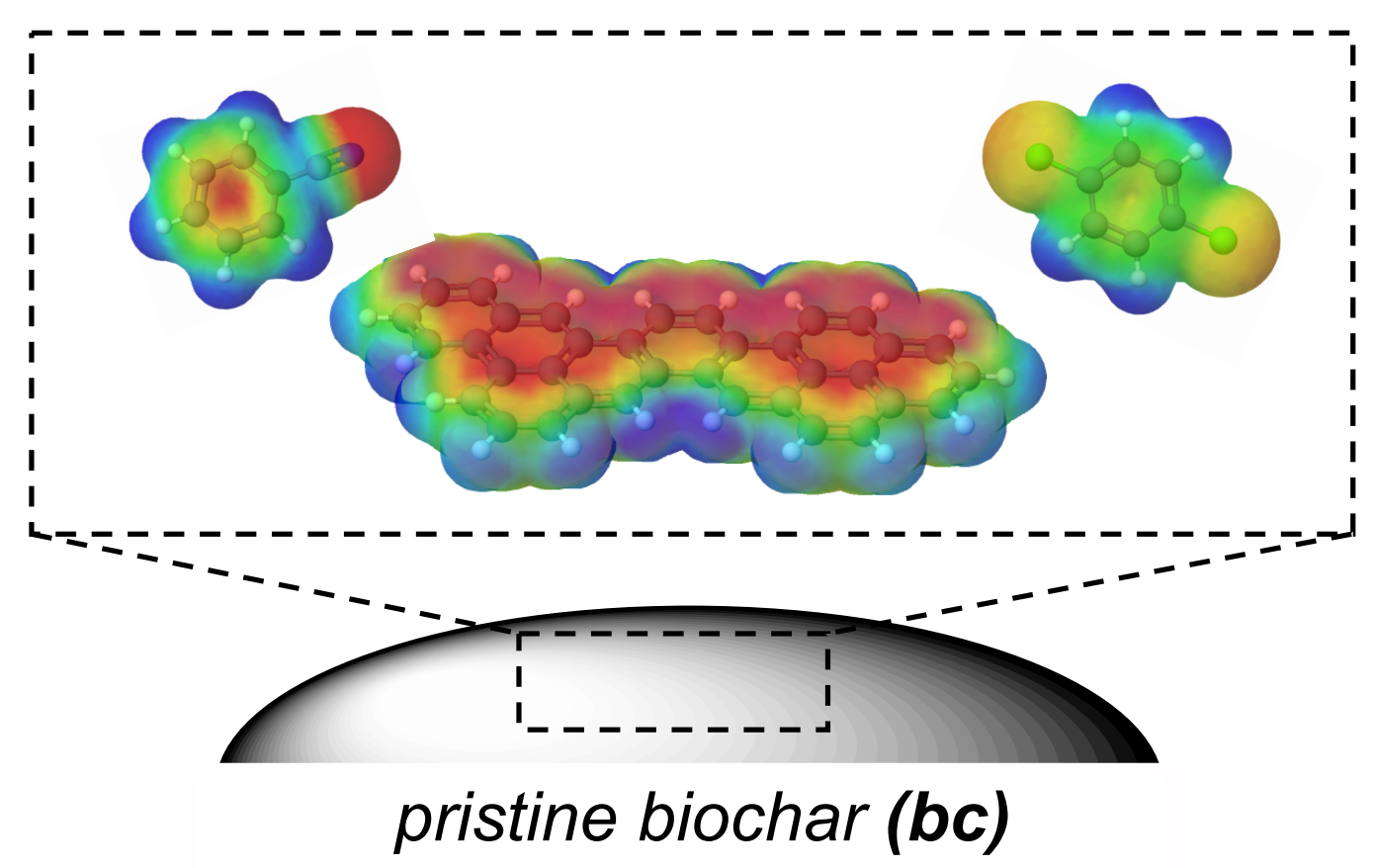

Figure 7 -Proposed interactions between bc and solvents with high polarizability.

Kamlet-Taft solvatochromic parameters are the most extensively used quantitative measure of solvent characteristics and solvent-solute interactions. ${ }^{26,46}$ They are divided into three: $\alpha, \beta$, and $\pi^{*}$. The parameter $\alpha$ quantifies the ability of the solvent to donate a hydrogen-bond, $\beta$ is a scale of its ability to accept a hydrogen-bond, and $\pi^{*}$ represents the solvent's polarizability. ${ }^{27}$ Most solvents present values between 0 and 1 on each scale. Although the Kamlet-Taft solvatochromic parameters have been widely utilized in the investigation of diverse solvent-solute systems, to the best of our knowledge, they have not been applied to explore LPE of materials and solvents used to stabilize the resulting nanostructures. For those reasons, we decided to determine if there was any correlation between the percentage of biochar dispersed in our samples and $\alpha, \beta$, and $\pi^{*}$ values of the solvents used. As shown in our preliminary studies and in Table S1 (Supporting Information), good dispersions of pristine biochar (bc) nanosheets could be obtained in solvents with $\rho \sim 1.0 \mathrm{~g} / \mathrm{mL}$ and $\gamma \sim$ $20 \mathrm{mN} / \mathrm{m}$. However, solvents such as PhCN, 1,2-DCB, NMP, and solketal, which present $\gamma$ values higher than $20 \mathrm{mN} / \mathrm{m}$ and out of the ideal range, could also successfully disperse pristine biochar samples. These results can be understood better by looking at the solvent's Kamlet-Taft solvatochromic parameters. The effective solvents for bc dispersion with non-ideal $\rho$ and $\gamma$ values presented $\pi^{*} \geq 0.50$. This observation indicated that solvatochromic parameters could actually help to explain the nature of biochar exfoliation, since solvents with high polarizability values (i.e. high values of $\pi^{*}$ ) could interact more effectively with the electron cloud generated by the graphene-like sheets within the biochar structure, as is shown pictorially in Figure 7.

LPE of oxidized biochar (ox-bc) was not effective in the same solvent that worked for bc exfoliation and was found to be dependent on different solvent parameters. From the results in Table S2 (Supporting Information), we determined that there were two classes of solvents that could produce ox-bc nanosheets via LPE: Solvents with $\rho \sim 1.0 \mathrm{~g} / \mathrm{mL}$ and $\gamma \sim 40$ $\mathrm{mN} / \mathrm{m}$ or solvents with $\beta$ values $\geq 0.50$ if the surface energies and densities were different to former. We propose that solvents with good hydrogen-bonding acceptance ability (i.e. high $\beta$ values) are able to interact more effectively with the hydroxyl and carboxyl groups on the surface of the oxidized biochar (Figure 8), and this correlates well with the increased acidity of the surface of ox-bc as characterized via Boehm titrations. 


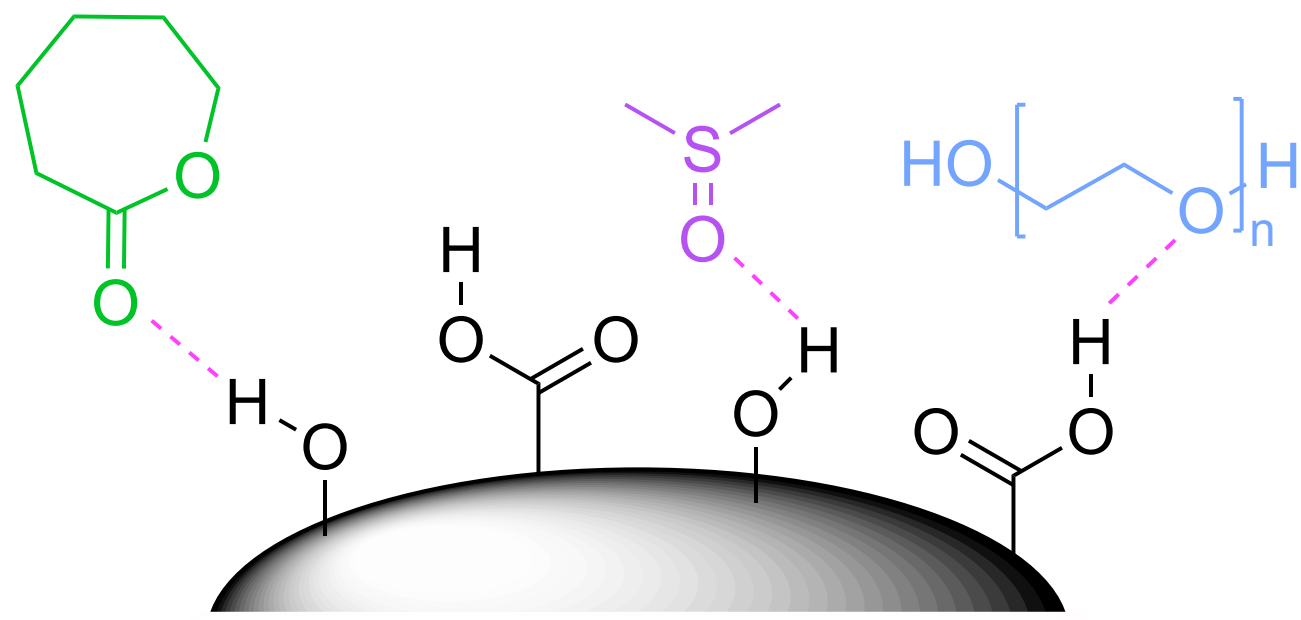

\section{oxidized biochar (ox-bc)}

Figure 8 - Proposed interactions between ox-bc and solvents with hydrogen-bonding acceptance ability.

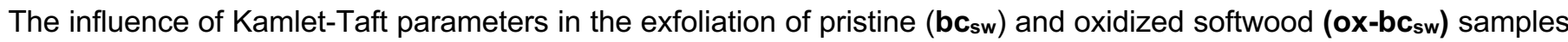
is summarized in plots shown in Figure 9. $\mathrm{H}_{2} \mathrm{O}$ and $\mathrm{C}_{6} \mathrm{~F}_{6}$ were excluded from this correlation due to their exceptional density and surface tension values. For pristine samples (Figure 9A), a synergic effect between the surface tension of the solvents $(\gamma)$ and their $\pi^{*}$ values is observed, and the highest levels of dispersion (orange region in graph) are obtained when solvents have low $\gamma$ and high $\pi^{*}$ values. For the oxidized samples (Figure 9B), an increase in exfoliation efficiency is seen with increasing values of $\gamma$ or $\beta$. Therefore, the dispersion of $\mathbf{o x}-\mathbf{b} \mathbf{c}_{\mathbf{s w}}$ is greatly increased when solvents with high values of $\gamma$ and $\beta$ are used for exfoliation (orange region in graph).
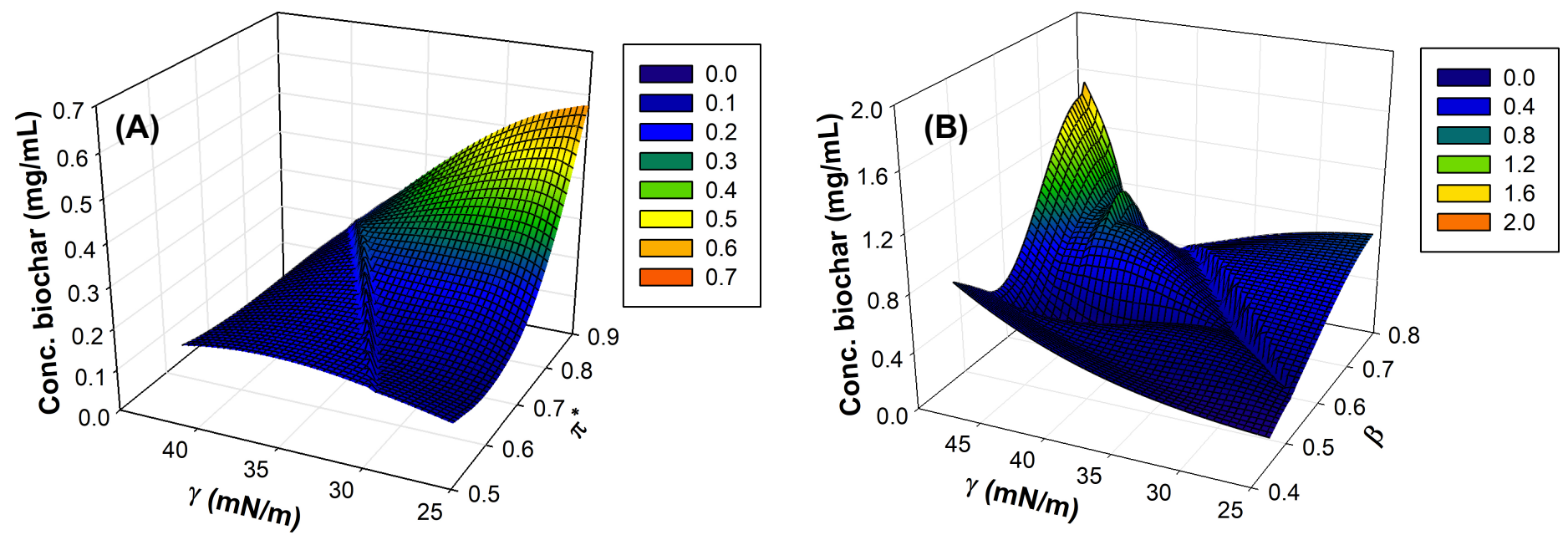

Figure 9 - Influence of $\pi^{*}, \beta$ and $\gamma$ values in the exfoliation of $(\mathrm{A}) \mathbf{b c}_{\mathbf{s w}}$ and $(\mathrm{B}) \mathbf{o x}-\mathbf{b} \mathbf{c}_{\mathbf{s w}}$ samples. $\mathrm{H}_{2} \mathrm{O}$ and $\mathrm{C}_{6} \mathrm{~F}_{6}$ have not been included in the data analysis due to their exceptional values of density and surface tension, respectively.

Although some of the high-performance solvents for the production of biochar nanostructures presented desirable characteristics for processing (e.g. high wt\% dispersed and low-boiling points), the applications of the exfoliated material would be limited due to the high toxicity of the solvents used. $\mathrm{CHCl}_{3}, \mathrm{CH}_{2} \mathrm{Cl}_{2}$, and 1,2-DCB are able to disperse more than $0.20 \mathrm{mg} / \mathrm{mL}$ of bc, but are toxic and suspected human carcinogens according to the International Agency for Research on Cancer (IARC). ${ }^{47-49}$ Solvents such as acetone, EtOAc, DMC, solketal, and even $\mathrm{H}_{2} \mathrm{O}$ can disperse about $0.10 \mathrm{mg} / \mathrm{mL}$ of bc 
using only 15 min sonication. These solvents are considered greener alternatives due to their low toxicity and high biodegradability, as indicated by various solvent selection guides. ${ }^{50-52}$ They also show similar results to the most commonly used solvent for biochar exfoliation, NMP. Moreover, $\mathrm{DMC}$ can also be derived from $\mathrm{CO}_{2}$, contributing to the storage, usage, and transformation of this greenhouse gas. ${ }^{52}$

Nanostructures of ox-bc could be obtained in solvents typically used for the LPE of layered materials, such as NMP and DMSO. However, in the current study, EG and PEGs 200 and 400 could yield more than $0.40 \mathrm{mg} / \mathrm{mL}$ of exfoliated oxbc due to their ideal surface tensions combined with their excellent hydrogen-bonding acceptance ability ( $\beta$ ). PEGs, although polymeric, provide excellent green liquid environments for LPE, because of their biodegradability, biocompatibility, non-toxicity, low flammability, and vapor pressure. ${ }^{53}$ PEGs are also widely used in consumer products, and are approved for internal consumption by the US Food and Drug Agency. ${ }^{53}$ EG can be considered a less desired alternative when compared to PEGs for the exfoliation of ox-bc, because high doses can be toxic to human and aquatic biota upon ingestion or exposure. ${ }^{54}$ However, EG can be produced sustainably from lignocellulosic biomass, bacteria, and algae. ${ }^{55}$ Although $\varepsilon^{-}$ $\mathrm{CL}$ and solketal have a lower surface tension value when compared to EG, PEG 200, and PEG 400, they can exfoliate more than $0.20 \mathrm{mg} / \mathrm{mL}$ of ox-bc. $\varepsilon-C L$ can cause eye irritation, but overall is considered a nontoxic, affordable, and important commodity chemical for the production of the biodegradable poly( $\varepsilon$-caprolactone $).{ }^{56}$ It is not widely used as an ester solvent unlike more volatile esters such as ethyl acetate.

A comparison of green and traditional solvents for LPE of bc and ox-bc is presented in Figure 10. Based on the diagram, we can observe that under the conditions explored, ox-bc generally achieves higher levels of dispersion $(0.75$ $\mathrm{mg} / \mathrm{mL}$ maximum) when compared to bc $(0.51 \mathrm{mg} / \mathrm{mL}$ maximum). This is likely due to the hydrogen-bond donating ability of the functional groups on the surface of ox-bc.
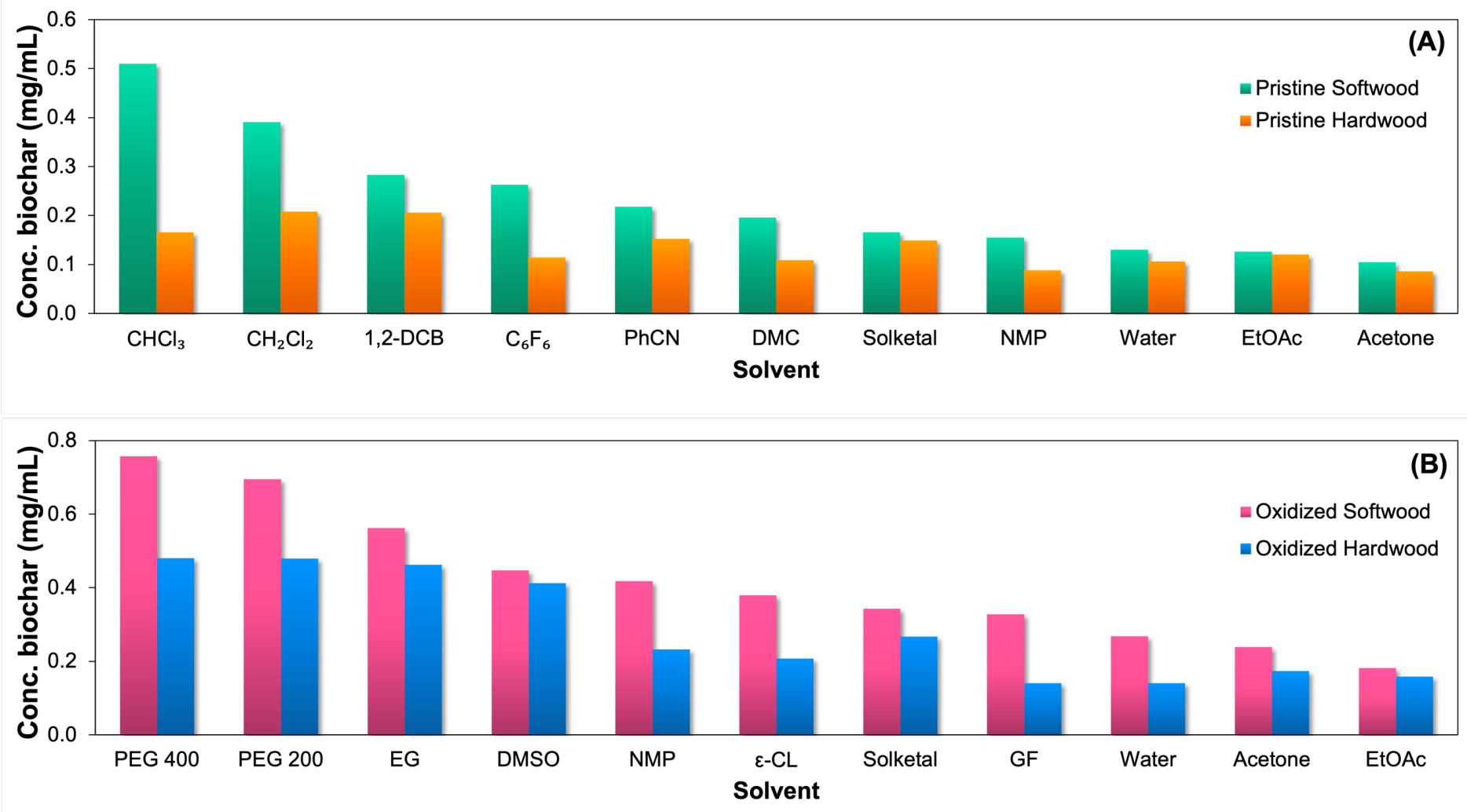

Figure 10 - Concentrations of exfoliated biochar dispersed in samples of (A) bc and (B) ox-bc in different solvents.

Effect of sonication time on LPE of biochar. After the investigation of greener environments for the LPE of biochar, we decided to evaluate the influence of time on the process, which has shown to be an important parameter in the exfoliation 
of graphene in a previous work. ${ }^{39}$ For those reasons, $\mathbf{b c}_{\mathrm{hw}}$ samples were sonicated for 15, 30, 60, 90, and 120 min in EtOAc. The procedure was performed in triplicate, and the concentration of $\mathbf{b c}_{\mathrm{hw}}$ dispersed in the samples was found to increase gradually, Figure 11.

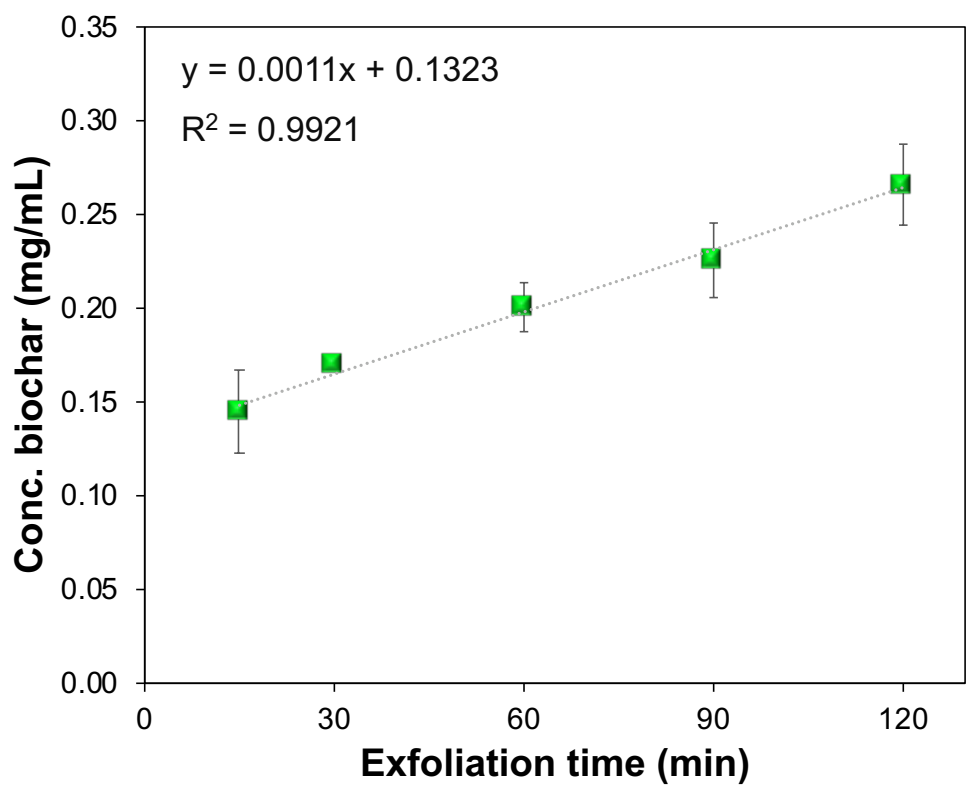

Figure 11 - Concentration of exfoliated $\mathbf{b} \mathbf{c}_{\mathrm{hw}}$ as a function of exfoliation time in ethyl acetate, showing a linear behavior and following the equation $\mathrm{y}=$ $0.0011 x+0.1323$

Although the yield of nanostructures dispersed in the medium increases with sonication time, the quality of the exfoliated material can decrease during longer LPEs. To avoid this problem, the use of viscous solvents is often required to stabilize and preserve the obtained dispersions. ${ }^{39}$ For those reasons, we assessed the quality of the produced biochar nanostructures after different exfoliation times via Raman spectroscopy, calculated $I_{G} / I_{D}$ ratios for each processing time (Figure 12). Interestingly, there was no significant decrease in the $I_{G} / l_{D}$ ratio values, indicating that better yields of biochar nanostructures can be obtained herein during longer sonication times even in solvents with low viscosities.

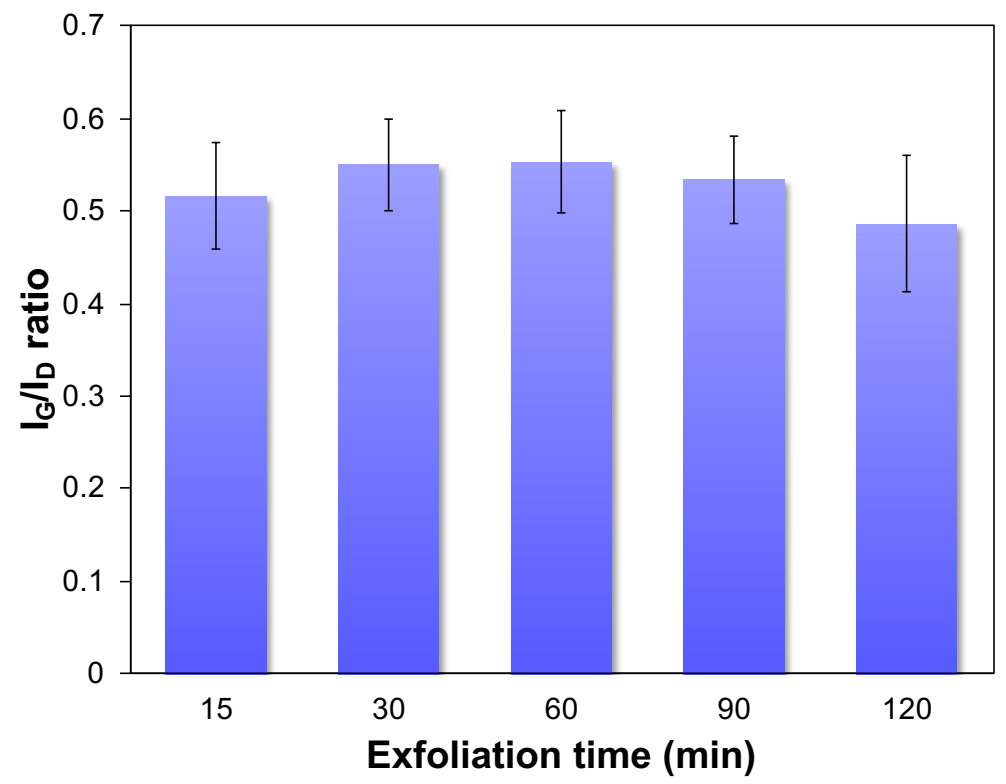

Figure $12-\mathrm{I}_{\mathrm{G}} / \mathrm{l}_{\mathrm{D}}$ ratios of exfoliated $\mathbf{b} \mathbf{c}_{\mathrm{hw}}$ as a function of processing time in ethyl acetate. 


\section{CONCLUSIONS}

LPE has been applied to pristine and functionalized biochars from different waste biomass feedstocks to obtain nanostructures of the respective layered materials and discover more benign environments for LPE. The nanostructures of exfoliated biochar obtained are comprised of a small number (i.e. 2-8) of stacked layers and have the potential to be applied in high value fields. During our investigation, both solvent surface-matching properties such as surface tension $(\gamma)$ and density $(\rho)$, and biochar-solvent intermolecular interactions have been shown to greatly influence the process. Although different solubility parameters were used with or without success to investigate LPE of other materials previously, to our knowledge, this is the first time that Kamlet-Taft solvatochromic parameters have been used to aid the understanding of this exfoliation method.

Solvents with $\rho \sim 1.0 \mathrm{~g} / \mathrm{mL}, \gamma \sim 20 \mathrm{mN} / \mathrm{m}$ or high values of $\pi^{*}$ showed good performance in the exfoliation of pristine biochars, whereas solvents with $\rho \sim 1.0 \mathrm{~g} / \mathrm{mL}, \gamma \sim 40 \mathrm{mN} / \mathrm{m}$ or with good hydrogen-bond accepting ability $(\beta)$ could successfully exfoliate oxidized biochars. Using only 15 min sonication, dispersions containing more than $0.10 \mathrm{mg} / \mathrm{mL}$ of pristine biochar could be obtained in solvents less harmful than NMP, which is typically used for LPE. These solvents include acetone, dimethyl carbonate, ethyl acetate, solketal, and deionized water. Significant dispersions of exfoliated oxidized biochar nanosheets (>0.20 mg/mL) could also be obtained in more benign solvents such as $\varepsilon$-caprolactone, ethylene glycol, and polyethylene glycols 200 and 400. Moreover, no strict control of temperature was needed, and no surfactants were used to stabilize the dispersions. These data and reasonings will hopefully allow others to evaluate the solvents applied in LPE of other materials, employing greener alternatives to commonly used toxic solvents. The produced biochar nanostructures in the current study have large potential to be applied as reinforcing agents in polymer composites, an investigation of which will be pursued in our future work.

\section{ACKNOWLEDGMENTS}

Financial support was provided by the Natural Sciences and Engineering Research Council (NSERC), Canada Foundation for Innovation (CFI), the provincial government of Newfoundland and Labrador, and Memorial University of Newfoundland (MUN). Dr. Kelly Hawboldt (Faculty of Engineering and Applied Science, MUN) is thanked for softwood biochar samples, Judy Maclnnis (Cape Breton University) is thanked for TEM micrographs, Dr. Erika Merschrod and Dr. Stefana Egli (Department of Chemistry, MUN) for valuable discussions.

Keywords: biomass, exfoliation, nanostructures, green solvents, green chemistry.

\section{REFERENCES}

1. J. Lehmann and S. Joseph, Biochar for environmental management: Science and Technology, Earthscan, Sterling, VA, 2009.

2. D. Woolf, J. E. Amonette, F. A. Street-Perrott, J. Lehmann and S. Joseph, Nature Communications, 2010, 1, 5664.

3. W. J. Liu, H. Jiang and H. Q. Yu, Chem. Rev., 2015, 115, 12251-12285.

4. P. Basu, in Biomass Gasification, Pyrolysis and Torrefaction (Second Edition), ed. P. Basu, Academic Press, Boston, 2013, pp. 147-176.

5. J. L. Vidal, V. P. Andrea, S. L. MacQuarrie and F. M. Kerton, ChemCatChem, 2019, 11, 4089-4095.

6. Z. Li, R. J. Young, C. Backes, W. Zhao, X. Zhang, A. A. Zhukov, E. Tillotson, A. P. Conlan, F. Ding, S. J. Haigh, K. S. Novoselov and J. N. Coleman, ACS Nano, 2020, 14, 10976-10985. 
J. N. Coleman, M. Lotya, A. O'Neill, S. D. Bergin, P. J. King, U. Khan, K. Young, A. Gaucher, S. De, R. J. Smith, I. V. Shvets, S. K. Arora, G. Stanton, H.-Y. Kim, K. Lee, G. T. Kim, G. S. Duesberg, T. Hallam, J. J. Boland, J. J. Wang, J. F. Donegan, J. C. Grunlan, G. Moriarty, A. Shmeliov, R. J. Nicholls, J. M. Perkins, E. M. Grieveson, K. Theuwissen, D. W. McComb, P. D. Nellist and V. Nicolosi, Science, 2011, 331, 568-571.

8. H. Tao, Y. Zhang, Y. Gao, Z. Sun, C. Yan and J. Texter, Phys. Chem. Chem. Phys., 2017, 19, 921-960.

9. G. Cunningham, M. Lotya, N. McEvoy, G. S. Duesberg, P. van der Schoot and J. N. Coleman, Nanoscale, 2012, 4, 6260-6264.

10. R. J. Smith, P. J. King, M. Lotya, C. Wirtz, U. Khan, S. De, A. O'Neill, G. S. Duesberg, J. C. Grunlan, G. Moriarty, J. Chen, J. Wang, A. I. Minett, V. Nicolosi and J. N. Coleman, Adv. Mater., 2011, 23, 3944-3948.

11. Y. Zhu, S. Murali, M. D. Stoller, K. J. Ganesh, W. Cai, P. J. Ferreira, A. Pirkle, R. M. Wallace, K. A. Cychosz, M. Thommes, D. Su, E. A. Stach and R. S. Ruoff, Science, 2011, 332, 1537-1541.

12. P. May, U. Khan, A. O'Neill and J. N. Coleman, J. Mater. Chem., 2012, 22, 1278-1282.

13. S. Pavlidou and C. D. Papaspyrides, Prog. Polym. Sci., 2008, 33, 1119-1198.

14. S. Stankovich, D. A. Dikin, G. H. B. Dommett, K. M. Kohlhaas, E. J. Zimney, E. A. Stach, R. D. Piner, S. T. Nguyen and R. S. Ruoff, Nature, 2006, 442, 282-286.

15. H.-D. Huang, P.-G. Ren, J. Chen, W.-Q. Zhang, X. Ji and Z.-M. Li, J. Membr. Sci., 2012, 409-410, 156-163.

16. V. Nicolosi, M. Chhowalla, M. G. Kanatzidis, M. S. Strano and J. N. Coleman, Science, 2013, 340, 12264191226437.

17. C. Backes, T. M. Higgins, A. Kelly, C. Boland, A. Harvey, D. Hanlon and J. N. Coleman, Chem. Mater., 2016, 29, 243-255.

18. Y. Hernandez, V. Nicolosi, M. Lotya, F. M. Blighe, Z. Sun, S. De, I. T. McGovern, B. Holland, M. Byrne, Y. K. Gun'Ko, J. J. Boland, P. Niraj, G. Duesberg, S. Krishnamurthy, R. Goodhue, J. Hutchison, V. Scardaci, A. C. Ferrari and J. N. Coleman, Nat. Nanotechnol., 2008, 3, 563-568.

19. N. Wang, Q. Xu, S. Xu, Y. Qi, M. Chen, H. Li and B. Han, Sci. Rep., 2015, 5, 16764-16772.

20. M. Lancaster, Green chemistry: An introductory text, RSC Publishing, 2002.

21. A. Ciesielski and P. Samori, Chem. Soc. Rev., 2014, 43, 381-398.

22. J. Shen, Y. He, J. Wu, C. Gao, K. Keyshar, X. Zhang, Y. Yang, M. Ye, R. Vajtai, J. Lou and P. M. Ajayan, Nano Lett., 2015, 15, 5449-5454.

23. S. D. Bergin, Z. Sun, D. Rickard, P. V. Streich, J. P. Hamilton and J. N. Coleman, ACS Nano, 2009, 3, 2340-2350.

24. C. Reichardt, in Solvents and Solvent Effects in Organic Chemistry, Wiley-VCH, Weinhem, 3rd edn., 2003, pp. 5-

56.

25. K.-G. Zhou, N.-N. Mao, H.-X. Wang, Y. Peng and H.-L. Zhang, Angew. Chem. Int. Ed., 2011, 50, 10839-10842.

26. P. G. Jessop, D. A. Jessop, D. Fu and L. Phan, Green Chem., 2012, 14, 1245-1259.

27. M. J. Kamlet, J. L. M. Abboud, M. H. Abraham and R. W. Taft, J. Org. Chem., 1983, 48, 2877-2887.

28. X. Xiao and B. Chen, Environ. Sci. Technol., 2017, 51, 5473-5482.

29. G. Liu, H. Zheng, Z. Jiang, J. Zhao, Z. Wang, B. Pan and B. Xing, Environ. Sci. Technol., 2018, 52, 10369-10379.

30. P. Oleszczuk, W. Ćwikła-Bundyra, A. Bogusz, E. Skwarek and Y. S. Ok, J. Anal. Appl. Pyrol., 2016, 121, 165-172.

31. W. Tian, Q. Gao, A. VahidMohammadi, J. Dang, Z. Li, X. Liang, M. M. Hamedi and L. Zhang, Chem. Eng. J., 2020, $127601-127612$.

32. M. Naghdi, M. Taheran, S. K. Brar, T. Rouissi, M. Verma, R. Y. Surampalli and J. R. Valero, J. Clean. Prod., 2017, 164, 1394-1405.

33. L. Li, K. Zhang, L. Chen, Z. Huang, G. Liu, M. Li and Y. Wen, New J. Chem., 2017, 41, 9649-9657. 
34. M. Genovese, J. Jiang, K. Lian and N. Holm, J. Mater. Chem. A, 2015, 3, 2903-2913.

35. S. Roy, U. Kumar and P. Bhattacharyya, Environ. Sci. Pollut. Res. Int., 2019, 26, 7272-7276.

36. A. J. Carrier, I. Abdullahi, K. A. Hawboldt, B. Fiolek and S. L. MacQuarrie, J. Phys. Chem. C, 2017, 121, 2630026307.

37. S. L. Goertzen, K. D. Thériault, A. M. Oickle, A. C. Tarasuk and H. A. Andreas, Carbon, 2010, 48, 1252-1261.

38. A. M. Oickle, S. L. Goertzen, K. R. Hopper, Y. O. Abdalla and H. A. Andreas, Carbon, 2010, 48, 3313-3322.

39. L. Xu, J.-W. McGraw, F. Gao, M. Grundy, Z. Ye, Z. Gu and J. L. Shepherd, J. Phys. Chem. C, 2013, 117, 1073010742.

40. H. J. Salavagione, J. Sherwood, M. De bruyn, V. L. Budarin, G. J. Ellis, J. H. Clark and P. S. Shuttleworth, Green Chem., 2017, 19, 2550-2560.

41. A. C. Ferrari and J. Robertson, Phys. Rev. B, 2000, 61, 14095-14107.

42. C. Guizani, K. Haddad, L. Limousy and M. Jeguirim, Carbon, 2017, 119, 519-521.

43. L. G. Cançado, A. Jorio, E. H. M. Ferreira, F. Stavale, C. A. Achete, R. B. Capaz, M. V. O. Moutinho, A. Lombardo, T. S. Kulmala and A. C. Ferrari, Nano Lett., 2011, 11, 3190-3196.

44. L. G. Cançado, K. Takai, T. Enoki, M. Endo, Y. A. Kim, H. Mizusaki, A. Jorio, L. N. Coelho, R. Magalhães-Paniago and M. A. Pimenta, Appl. Phys. Lett., 2006, 88, 163106-163109.

45. C. M. Hansen, Hansen Solubility Parameters: A User's Handbook, CRC Press: Taylor \& Francis, Boca Raton, 2nd edn., 2012.

46. L. Crowhurst, R. Falcone, N. L. Lancaster, V. Llopis-Mestre and T. Welton, J. Org. Chem., 2006, 71, 8847-8853.

47. IARC monographs on the evaluation of carcinogenic risks to humans: Chloroform, IARC, Lyon, France, 1999.

48. IARC monographs on the evaluation of carcinogenic risks to humans: Dichlorobenzenes, IARC, Lyon, France, 1999.

49. IARC monographs on the evaluation of carcinogenic risks to humans: Dichloromethane, IARC, Lyon, France, 2018.

50. C. M. Alder, J. D. Hayler, R. K. Henderson, A. M. Redman, L. Shukla, L. E. Shuster and H. F. Sneddon, Green Chem., 2016, 18, 3879-3890.

51. K. Alfonsi, J. Colberg, P. J. Dunn, T. Fevig, S. Jennings, T. A. Johnson, H. P. Kleine, C. Knight, M. A. Nagy, D. A. Perry and M. Stefaniak, Green Chem., 2008, 10, 31-36.

52. D. Prat, A. Wells, J. Hayler, H. Sneddon, C. R. McElroy, S. Abou-Shehada and P. J. Dunn, Green Chem., 2016, 18, 288-296.

53. F. M. Kerton and R. Marriott, in Alternative Solvents for Green Chemistry (Second Edition), The Royal Society of Chemistry, Cambridge, 2013, pp. 242-261.

54. C. A. Staples, J. B. Williams, G. R. Craig and K. M. Roberts, Chemosphere, 2001, 43, 377-383.

55. C. J. Clarke, W. C. Tu, O. Levers, A. Brohl and J. P. Hallett, Chem. Rev., 2018, 118, 747-800.

56. M. Labet and W. Thielemans, Chem. Soc. Rev., 2009, 38, 3484-3504. 